Orthopäde $2011 \cdot 40: 5-5$

DOI 10.1007/s00132-010-1673-6

Online publiziert: 12. Dezember 2010

(c) Springer-Verlag 2010

\author{
M. Scheibel \\ Centrum für Muskuloskeletale Chirurgie (CMSC), Charité-Universitätsmedizin Berlin, \\ Campus Mitte und Campus Virchow-Klinikum, Berlin
}

\title{
Aktuelle Entwicklungen in der arthroskopischen Schulterchirurgie
}

der knöchernen Rotatorenmanschettenausrisse trifft dies v. a. auf die arthroskopischen Knochenblockverfahren zur Behandlung knöchern bedingter Schulterinstabilitäten zu. Die jüngst beschriebenen arthroskopischen Techniken zur Behandlung von Nervenkompressionssyndromen der Schulter, die Möglichkeiten der arthroskopischen Entfernung verschiedenster Osteosynthesematerialien und der Einsatz neuer arthroskopischer Verfahren bei Knorpeldefekten des Schultergelenks spiegeln des Weiteren die andauernde und fast schon revolutionäre Entwicklung auf diesem Gebiet wider. Möglich war dies insbesondere durch das zunehmende Verständnis der arthroskopischen Anatomie bzw. Pathomorphologie und die Neubzw. Weiterentwicklung von Implantaten und Instrumenten, wodurch gegenwärtig selbst komplexe rekonstruktive Eingriffe arthroskopisch standardisiert und mit deutlich reduzierter Zugangsmorbidität durchgeführt werden können.

Ziel dieses Themenheftes ist es, Ihnen einen Überblick zu den aktuellsten Entwicklungen in der arthroskopischen Chirurgie des Schultergelenks und -gürtels zu geben. Die kommenden Jahre werden weitere Innovationen und Techniken auf diesem Gebiet mit sich bringen, und es ist und wird unsere Aufgabe sein, die Möglichkeiten und Grenzen des arthroskopischen Handelns neu zu definieren. Dennoch ist es sicherlich nicht übertrieben, heute zu behaupten, dass das Arthroskop in den Händen des Geübten nicht mehr das ,Werkzeug des Teufels“, sondern vielmehr ein „Geschenk Gottes“ darstellt.
In diesem Sinne wünsche ich Ihnen viel Freude bei der Durchsicht des vorliegenden Themenheftes.

Ihr

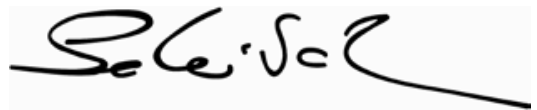

M. Scheibel

\section{Korrespondenzadresse}

PD Dr. M. Scheibel

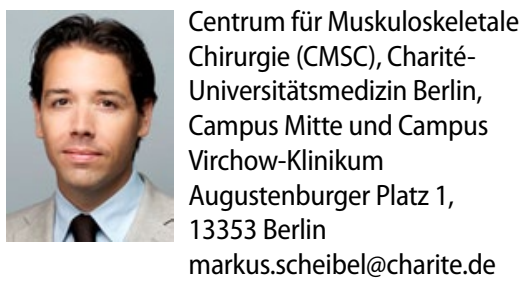

